\title{
Cerebrospinal Fluid Concentrations of Neuronal Proteins Are Reduced in Primary Angiitis of the Central Nervous System
}

\author{
Tillmann Ruland ${ }^{1,2}$, Jolien Wolbert ${ }^{1}$, Michael G. Gottschalk ${ }^{3}$, Simone König ${ }^{4}$, \\ Andreas Schulte-Mecklenbeck ${ }^{1}$, Jens Minnerup ${ }^{1}$, Sven G. Meuth ${ }^{1}$, Catharina C. Groß ${ }^{1}$, \\ Heinz Wiendl ${ }^{1 *}$ and Gerd Meyer zu Hörste ${ }^{1}$ \\ ${ }^{1}$ Department of Neurology, University of Münster, Münster, Germany, ${ }^{2}$ Department of Psychiatry and Psychotherapy, \\ University of Münster, Münster, Germany, ${ }^{3}$ Department of Psychiatry and Psychotherapy, Medical Center - University of \\ Freiburg, Faculty of Medicine, University of Freiburg, Freiburg, Germany, ${ }^{4}$ Core Unit Proteomics, Interdisciplinary Center for \\ Clinical Research, University of Münster, Münster, Germany
}

OPEN ACCESS

Edited by:

Fabienne Brilot,

University of Sydney, Australia

Reviewed by:

Rishein Gupta,

University of Texas at San Antonio,

United States

Anne-Katrin Pröbstel,

University of California, San Francisco,

United States

*Correspondence: Heinz Wiend

heinz.wiendl@ukmuenster.de

Specialty section:

This article was submitted to

Multiple Sclerosis and

Neuroimmunology,

a section of the journal

Frontiers in Neurology

Received: 28 December 2017

Accepted: 17 May 2018

Published: 05 June 2018

Citation:

Ruland T, Wolbert J, Gottschalk MG König S, Schulte-Mecklenbeck A, Minnerup J, Meuth SG, Groß CC, Wiendl $H$ and Meyer zu Hörste $G$

(2018) Cerebrospinal Fluid

Concentrations of Neuronal Proteins Are Reduced in Primary Angiitis of the

Central Nervous System.

Front. Neurol. 9:407.

doi: 10.3389/fneur.2018.00407
Primary angiitis of the central nervous system (PACNS) is a rare autoimmune vasculitis limited to the CNS often causing substantial disability. Understanding of this disease is impaired by the lack of available biomaterial. Here, we collected cerebrospinal fluid (CSF) from patients with PACNS and matched controls and performed unbiased proteomics profiling using ion mobility mass spectrometry to identify novel disease mechanisms and candidate biomarkers. We identified 14 candidate proteins, including amyloid-beta A4 protein (APP), with reduced abundance in the CSF of PACNS patients and validated APP by Enzyme-linked Immunosorbent Assay (ELISA) in an extended cohort of patients with PACNS. Subsequent functional annotation surprisingly suggested neuronal pathology rather than immune activation in PACNS. Our study is the first to employ mass spectrometry to local immune reactions in PACNS and it identifies candidates such as APP with pathogenic relevance in PACNS to improve patient care in the future.

Keywords: PACNS, CSF, mass spectrometry, flow cytometry, amyloid-beta A4 protein

\section{INTRODUCTION}

Primary angiitis of the central nervous system (PACNS) is a rare form of vasculitis with approximately 700 reported cases published in the literature worldwide (1). PACNS is generally considered to be an autoimmune disease of the vasculature limited to vessels of the central nervous system (CNS). The pathogenesis of PACNS is poorly understood and therapy design is often challenging. Management of PACNS would be facilitated by improved diagnostic or prognostic biomarkers and mechanistic understanding of the disease. Unbiased screening approaches as a strategic could serve as the conceptual basis for subsequent mechanistic studies.

The median age at onset of PACNS is around 50 years and men are more often affected then women. Clinical presentation displays a considerable heterogeneity. Initially, PACNS patients may present with unspecific symptoms such as headache and encephalopathy. Fever, seizures or focal symptoms are less common, but can also occur. Moreover, strokes, transient ischaemic attacks, and hemorrhages occur in $30-50 \%$ of patients (2). Very little is known about the pathomechanisms of PACNS. Due to its clinical heterogeneity, different molecular subtypes of PACNS have been 
proposed encompassing granulomatous angiitis of the CNS and amyloid- $\beta$-related cerebral angiitis (ABRA) (2).

Histopathological studies have shown three distinct morphological patterns: acute necrotizing, purely lymphocytic, and granulomatous vasculitis (3). Histologically acute neutrophilic inflammation is the main diagnostic feature in active necrotizing vasculitis. Lymphocytic vasculitis features infiltration of $\mathrm{B}$ cells and granulomatous vasculitis is characterized by perivascular infiltration of mononuclear and granulomatous as well as multinucleated giant cells. Recent studies have claimed PACNS to be a $\mathrm{CD} 4^{+} \mathrm{T}$ cell-mediated disease. In fact, the CSF of a patient with ABRA contained a substantial fraction of activated $\mathrm{CD} 69^{+} \mathrm{CD} 4^{+} \mathrm{T}$ cells, even remote from the primary site of inflammation. $\mathrm{CD} 4^{+} \mathrm{T}$ cells were present in close proximity to MHC class II-expressing epithelioid macrophages and multinucleated giant cells (4). Furthermore, $\mathrm{CD}^{+} \mathrm{T}$ cells in PACNS produce significantly more pro-inflammatory interleukin-17 compared to ischemic stroke controls (5). Moreover, in ABRA CD $68^{+}$macrophages extend to the parenchyma surrounding the vessel walls (6). Nevertheless, the cause of PACNS remains unknown.

Present diagnostic criteria of PACNS include (1) the presence of an acquired otherwise unexplained neurological or psychiatric deficit, (2) the presence of either classic angiographic or histopathological features of angiitis within the CNS, and (3) no evidence of systemic vasculitis or any disorder that could cause or mimic the angiographic or pathological features of the disease (7).

Conventional CSF analysis in PACNS usually features lymphocytic pleocytosis and elevated protein levels and can thus aid in the diagnosis of PACNS. Magnetic resonance (MR) imaging can reveal ischemic and hemorrhagic lesions in cortical, subcortical, and deep gray matter structures (8). CNS angiography shows alterations of blood vessel irregularities such as stenosis and dilatation. Indirect non-invasive imaging angiography such as computed tomography (CT)- or MRangiography constitute alternatives to invasive CNS angiography. Recent advances in high resolution MR imaging of the vessel wall, such as black-blood contrast-enhanced $T 1$ weighted images, may also be useful in the assessment of PACNS (9-12). Finally, brain biopsy provides further important histopathological evidence for PACNS. However, none of the diagnostic procedures provide an easily quantifiable laboratory surrogate marker.

In summary, due to the small number of cases and limited amount of available biomaterial (e.g., blood, CSF, biopsy specimen) it has been difficult to identify molecular mechanisms to better understand the immunological signatures of PACNS and to articulate hypotheses regarding pathomechanisms. High throughput and unbiased proteomics may provide new molecular insights in rare diseases (13-15). Mass-spectrometrybased techniques allow determining the relative abundance of a considerable fraction of the proteome without a pre-selection of target proteins. Recent studies in neuropsychiatric disorders and cancer serve as an example and identified potential diagnostic and prognostic markers in serum $(16,17)$.

Here, we analyzed CSF cells by flow cytometry and noncellular CSF using ultrahigh-definition mass spectrometry followed by multivariate statistics in patients diagnosed with PACNS in comparison to a control group (18). Functional annotation network analysis was performed to identify relevant protein interactions and derive information of potentially affected biological pathways. Surprisingly, we find that APP, as validated by Enzyme-linked Immunosorbent Assay (ELISA), and other proteins of neuronal origin are less abundant in the CSF of PACNS patients suggesting a potential link between inflammation and neurodegeneration in this primary vasculitis as a conceptual basis for further mechanistic studies.

\section{MATERIALS AND METHODS}

\section{Study Participants and Sample Collection}

This study was approved by the ethics committee of the medical association Westphalia-Lippe, Germany (2016-053-f-S). Four patients diagnosed with PACNS were included. Diagnosis was based on clinical symptoms, MRI and angiography findings and after having ruled out systemic vasculitis. The imaging diagnosis of PACNS was based on digital subtraction angiography and MR imaging using black-blood contrast-enhanced T1 weighted sequences, which showed contrast enhancement in the vessel walls (see Figure 1). Four patients diagnosed with idiopathic intracranial hypertension (IIH) were chosen as matched controls (see Tables 1, 2 for details). Matching was performed for age, gender and BMI. Blood and CSF of all patients were routinely analyzed to rule out autoimmune, infectious, or malignant disease (see Table 2 for details).

The patients were followed-up in our department for at least 9 months after diagnosis was made. During this period consecutive MR imaging was performed and due to the diseases activity immunosuppressant therapy was started in all patients.

In addition, all CSF cell samples and matching blood samples were analyzed by flow cytometry using a predefined staining panel (see below) and the non-cellular CSF fraction was processed for consecutive proteome analysis. For this, CSF samples were centrifuged at $350 \mathrm{~g}$ for $10 \mathrm{~min}$, the CSF supernatant was removed and stored at $-80^{\circ} \mathrm{C}$ until further proteomics processing. Maximum storage time for any sample was 21 months with an average of $11.38 \pm 5.50$ months. For ELISA analysis an additional four patients diagnosed as described above as well as four controls with IIH were selected from our biobank (see Supplementary Table S4 for details).

\section{Flow Cytometry}

CSF and EDTA blood samples were obtained from all patients and processed as described previously (19). Briefly, cells from CSF or $100 \mu \mathrm{l}$ EDTA blood were incubated in VersaLyse buffer (Beckman Coulter; Brea, CA) for $10 \mathrm{~min}$ and subsequently washed three times with PBS supplemented with $2 \%$ heat-inactivated FCS and 2 mM EDTA. Following incubation with fluorochrome-conjugated antibodies (CD14-FITC, CD138PE, HLA-DR-ECD, CD3-PC5.5, CD56-PC7, CD4-APC, CD19APCAlexafluor700, CD16-APCAlexafluor750, CD8-PacificBlue, and CD45-KromeOrange, all Beckman Coulter), cells were acquired on a Navios flow cytometer (Beckman Coulter). Analysis was conducted with Kaluza V1.2. Lymphocytes, 

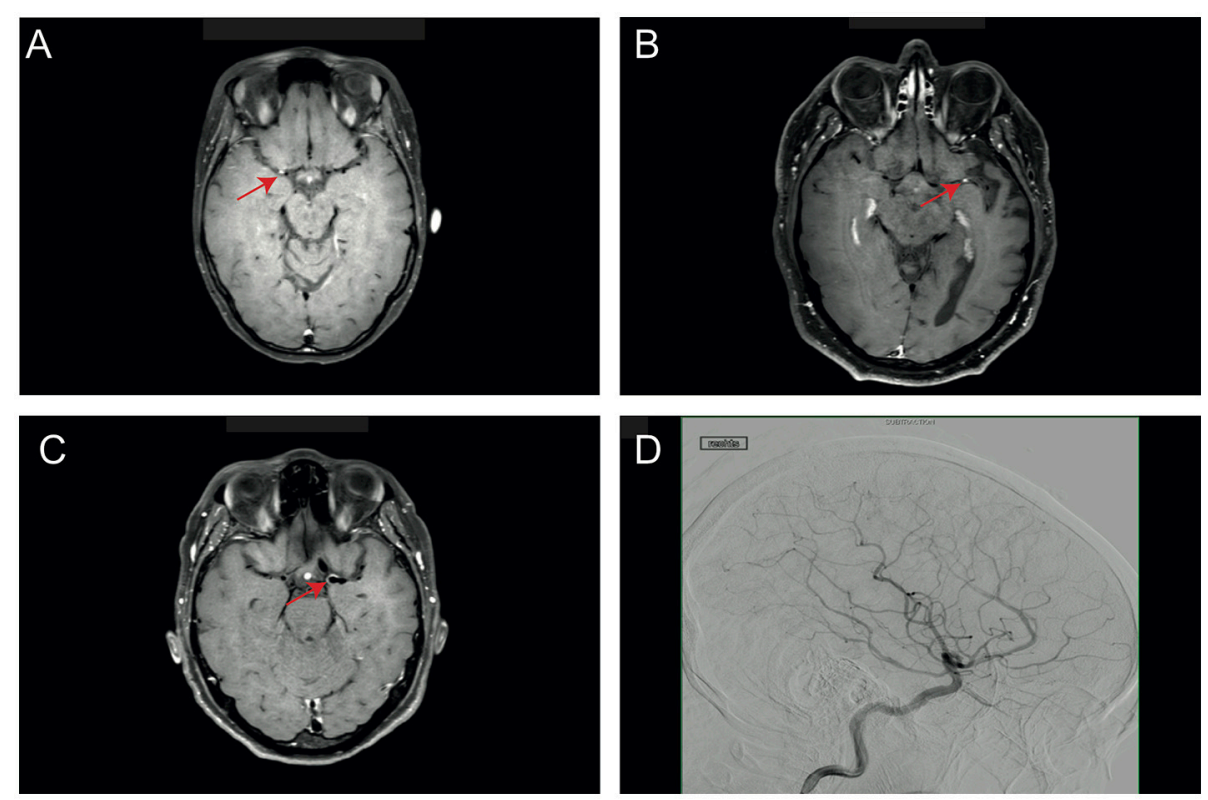

FIGURE 1 | Representative imaging findings in PACNS patients. (A-C) Images of patients 1-3 with black-blood contrast-enhanced $T 1$ weighted sequences, which show contrast enhancement in the vessel walls (red arrow). (D) Angiography image of PACNS patient 4 with longest duration of diseases showing pathognomonic blood vessel irregularities such as stenosis.

monocytes, and granulocytes were selected based on forwards scatter channel, sideward scatter channel, CD14, and CD45 expression characteristics. Lymphocytes subsets were selected as $\mathrm{CD}^{+}{ }^{+} \mathrm{CD} 4^{+}$( $\mathrm{T}$ helper cells), $\mathrm{CD}^{+} \mathrm{CD}^{+}$(cytotoxic $\mathrm{T}$ cells), $\mathrm{CD}^{+}{ }^{+} \mathrm{HLA}_{-} \mathrm{DR}^{+}$(activated $\mathrm{T}$ cells), $\mathrm{CD}^{-}{ }^{-} \mathrm{CD}^{+} 6^{+}$(NK cells), $\mathrm{CD}^{-}{ }^{-} \mathrm{CD} 19^{+}$(B cells), $\mathrm{CD}^{-} \mathrm{CD} 19^{+} \mathrm{CD} 138^{+}$(plasma cells), whereas monocyte subsets were selected as $\mathrm{CD} 14^{+} \mathrm{CD} 16^{-}$ (classical monocytes), $\mathrm{CD} 14^{+} \mathrm{CD} 16^{+}$(non-classical monocytes) cells.

\section{Liquid-Chromatography Ultra-High Definition Mass Spectrometry (LC-UDMSe) Analysis}

For proteome analysis, CSF samples (1 ml each) were depleted using a ProteoMiner Small Capacity Protein Enrichment Kit from Bio-Rad (Hercules, CA) according to the instructions of the manufacturer. For the second depletion, the eluate of the first depletion was re-buffered using Zeba-Spin desalting columns 7 $\mathrm{kD}$ (ThermoFisher) and the ProteoMiner beads were washed using $20 \%$ ethanol. The second depletion then proceeded in the same way as the first.

On filter-digestion and liquid-chromatography mass spectrometry (LC-MS) was performed as described previously (18). Briefly, the protein solution was transferred to Nanosep Omega filter units (10 kDa MWCO, Pall). The sample on the filter was rinsed by centrifuging $\left(14,000 \times \mathrm{g}, 4{ }^{\circ} \mathrm{C}, 15 \mathrm{~min}\right)$ with urea buffer (100 $\mu$ l, $8 \mathrm{M}$ urea, $100 \mathrm{mM}$ Tris Base, $\mathrm{pH}$ 7.5). For reduction, the sample was vortexed with $100 \mathrm{ml}$ urea buffer containing $50 \mathrm{mM}$ DTT for $30 \mathrm{~min}$ followed by centrifuging and washing with urea buffer as above. Alkylation proceeded for $25 \mathrm{~min}$ in urea buffer containing $50 \mathrm{mM}$ iodoacetamide $(100 \mu \mathrm{l})$ on a vortexer in the dark followed by centrifuging and incubation with $50 \mathrm{mM}$ DTT containing urea buffer $(100 \mu \mathrm{l})$ for $15 \mathrm{~min}$. Subsequently, the filter unit was rinsed with $100 \mu \mathrm{l}$ urea buffer followed by five times washing with $100 \mu \mathrm{l} 50 \mathrm{mM}$ ammonium bicarbonate in 5\% acetonitrile. For digestion, $40 \mu \mathrm{l}$ trypsin solution (40 $\mathrm{ng} / \mu \mathrm{l}$ in $5 \%$ acetonitrile) and $60 \mu \mathrm{l}$ mass spectrometry-grade water were added. The filter unit was covered with parafilm and tryptic digestion proceeded at $37^{\circ} \mathrm{C}$ overnight. Peptides were collected by centrifuging three times with $100 \mu \mathrm{l}$ $0.1 \%$ formic acid containing $5 \%$ acetonitrile; all solutions were pooled and dried. For analysis, peptides were re-dissolved in $100 \mu \mathrm{l}$ of the same solution. High-definition MS was performed using Synapt G2 Si ion mobility mass spectrometer coupled to M-Class ultra-performance liquid chromatography (UPLC) (Waters Corp.) with a 90 min gradient (solvent system $100 \%$ water vs. $100 \%$ acetonitrile, both containing $0.1 \%$ formic acid; trap column V/M Symmetry C18 $100 \AA 5 \mu \mathrm{m}, 180 \mu \mathrm{m} \times 20 \mathrm{~mm}$; reversed phase column HSS T3 $1.8 \mu \mathrm{m} 75 \mu \mathrm{m} \times 200 \mathrm{~mm} ; 0.5 \mu \mathrm{l}$ injection volume).

\section{Enzyme-Linked Immunosorbent Assay (ELISA)}

APP concentrations in the undepleted CSF samples were measured using a Human APP DuoSet ELISA kit from R\&D Systems, Minneapolis USA, according to the manufacturer's instructions with a minimum detection limit of $0.625 \mathrm{ng} / \mathrm{ml}$ and using a protein standard curve for quantification (see Supplementary Table S5 for details). 

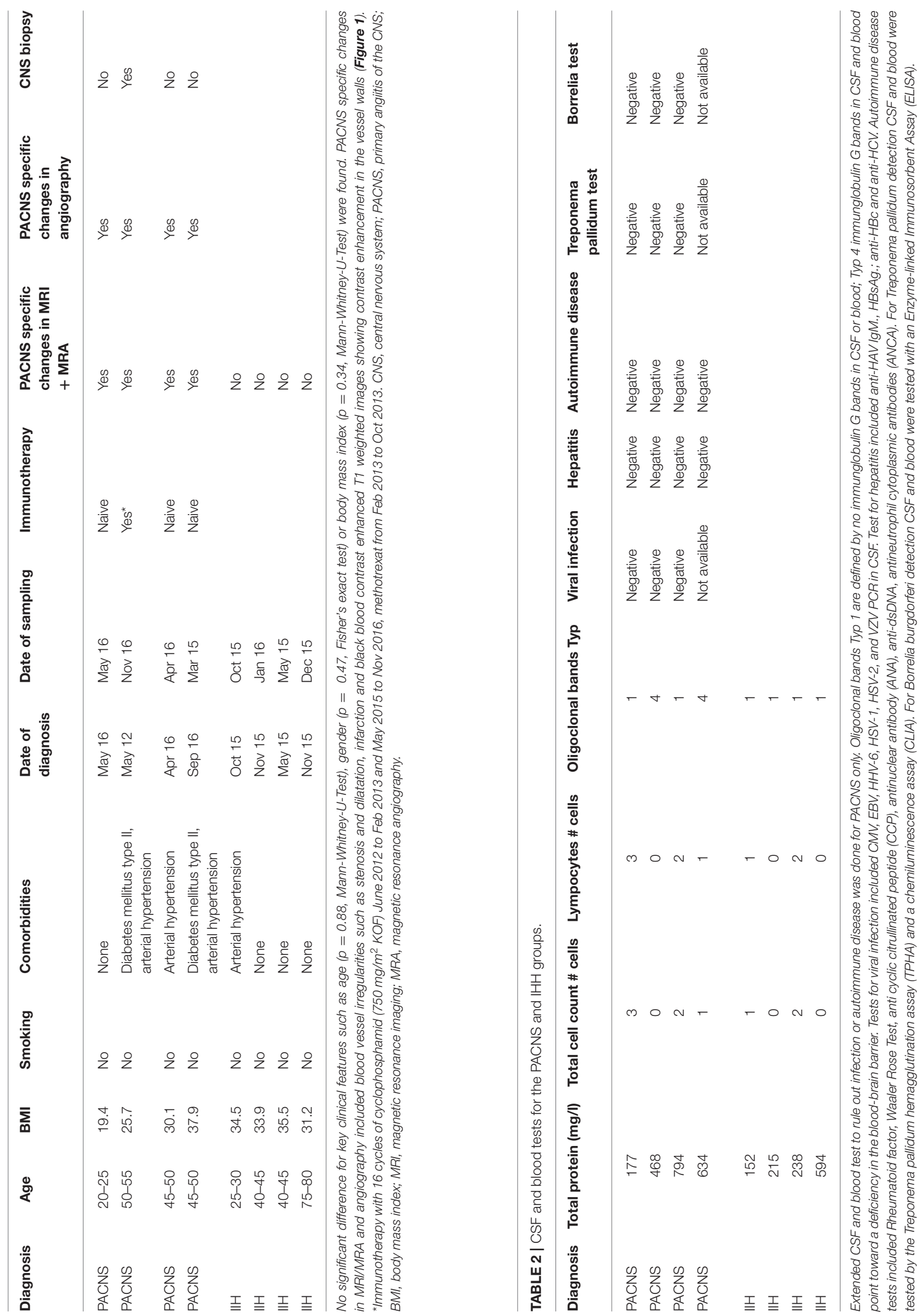


\section{Statistical Analysis of LC-UDMSe Data}

Data was pre-processed using Progenesis QI proteomics software (Nonlinear Dynamics,Version 2.0) using the UniProt human database (downloaded October 2015) generating normalized protein abundances for each sample.

Statistical analyses were additionally performed in R (http:// www.R-project.org/) (20). The Reproducibility Optimized Test Statistic (ROTS) R package version 1.6.0 was used for statistical analysis. ROTS aims to rank genomic features of interest (such as genes, proteins and transcripts) in order of evidence for differential expression in two-group comparisons. It uses a data adaptive method, which can optimize its parameters based on intrinsic features of input data. False discovery rate (FDR) was set below a value of $0.05(21,22)$. To increase confidence only proteins with $\geq 1$ unique peptide were considered for further analysis and were reported. A unique peptide is defined as a peptide, which based on its molecular properties, only exists in one protein and is therefore specific (23).

\section{Network Analysis}

A list of all proteins with fold changes found to be significantly different in the vasculitis vs. IIH comparison were uploaded to http://www.networkanalyst.ca/faces/home.xhtml. Using this online resource, a IMEx interactome based on the InnateDB platform was created (24). The network is generated by first mapping the significant proteins to the underlying proteinprotein interaction (PPI) database. A search algorithm is then performed to identify first-order neighbors (proteins that directly interact with a given protein) for each of these mapped proteins. The resulting nodes and their interaction partners are returned to build a subnetwork. Subsequently, a minimum network, which consisted of proteins directly interacting with each other, was chosen to study key nodes of functional connectivity $(25,26)$.

\section{Statistical Analysis of Flow Cytometry and ELISA Data}

After testing for homogeneity of variance and normal distribution flow cytometry data was analyzed using MannWhitney-U-Tests. The ELISA results were analyzed using a Student's $t$-test. Results were considered significant at a $p$-value threshold of $<0.05$.

\section{RESULTS}

We retrospectively identified four patients with PACNS from our inpatient clinic (Table 1), who were diagnosed based on clinical symptoms, MRI and angiography findings and after having ruled out systemic vasculitis (Table 2). We used four patients with IIH as control patients. All CSF samples had been analyzed by standard techniques. CSF supernatants had been collected and stored from all patients in a standardized fashion (see methods).

We compared demographic and clinical variables between groups (Tables 1, 2) and found no significant difference for age $(p=0.88)$, gender $(p=0.47)$, and body mass index $(p=0.34)$. Only one patient had been previously treated with two immunosuppressants and diagnosis had been established 54 months before sampling (see Table 1). For the other patients sampling was done at the time of PACNS diagnosis. One patient displayed symptoms of PACNS before diagnosis was made and sampling was performed at the onset of symptoms but before the diagnosis was established subsequently (Table 1).

We collected data of flow cytometry performed using cells from CSF and blood from all 8 patients in our study. Patients with PACNS exhibited a reduced percentage of $\mathrm{CD}^{+} \mathrm{T}$ cells in the peripheral blood compared to IIH patients $(p=0.029$, Figure 2C) but did not show any other alterations in the analyzed populations in blood or CSF (Supplementary Table S2), which suggests that in our small sized cohort CSF and blood do not exhibit distinct alterations in cell composition in PACNS. We therefore speculated that non-cellular immune mechanisms could also be relevant in PACNS. To identify such potential non-cellular changes in the CSF, we performed unbiased, mass spectrometry of the non-cellular fraction of CSF from PACNS and IIH patients.

First we depleted CSF samples of most abundant proteins to improve the sensitivity for detecting low abundance proteins. Then we performed non-hypothesis driven label-free liquidchromatography ultra-high definition mass spectrometry (LCUDMSe) analysis comparing the CSF of four patients with PACNS to four controls with IIH. We detected 591 quantifiable proteins in all CSF samples (Supplementary Table S1). We then tested for differential abundance between IIH and PACNS CSF samples and identified 95 proteins with differential abundance in PACNS-derived CSF $(p<0.05)$. To reduce the false positive rate, we applied very stringent selection criteria. After FDR correction, 14 proteins remained significantly different in PACNS (adjusted $p<0.05$ ) (Figure 2A). Notably, the vast majority of proteins was less abundant in PACNS samples. Except for CD62L/Lselectin (encoded by $S E L L$ ) detected proteins were related to neuronal or nervous system function rather than immune system function illustrated by proteins such as brain abundant membrane attached signal protein 1 (gene BASP1), amyloid beta A4 protein (gene $A P P$ ), and 14-3-3 proteins (genes $Y W H A B$ and $Y W H A H)$. This indicates that our screening approach preferentially detected abundance changes in proteins indicative of nervous system damage rather than reflecting immune cell activation or infiltration into the CSF compartment. Compared to candidates identified by mass spectrometry analysis of CSF in multiple sclerosis (MS) and Alzheimer's disease (AD) as well as human brain extracellular fluids in acute stroke overlap was modest. Only APP was also discovered to be changed in MS and AD (27-29).

Next, we aimed to validate the findings of our unbiased screen using an additional protein quantification method. We therefore quantified APP protein concentration by ELISA in CSF samples of an extended cohort of PACNS and control patients. We added four additional patients diagnosed with PACNS and four controls to the samples analyzed by LCUDMSe (Supplementary Tables S3, S4 for patients details). We found a significant decrease of APP concentrations in CSF in PACNS patients $(451.44 \pm 196.21 \mathrm{ng} / \mathrm{ml})$ vs. controls $(1513.7$ $\pm 255.55 \mathrm{ng} / \mathrm{ml}$ ); $t_{(14)}=5.61, p=0.0000641$ (Figure 2D). This supports the adequacy of our screening approach.

Next, we sought to embed proteins with differential abundance into a PPI network to aid our understanding of our findings. This PPI network analysis is based on publicly available 


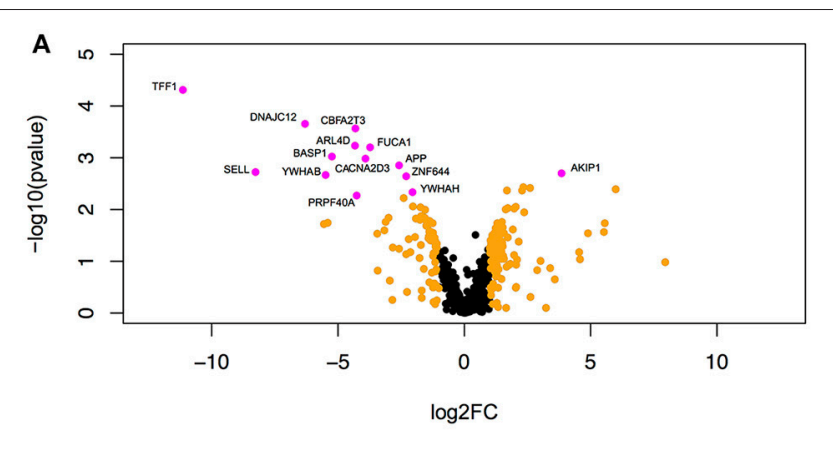

B

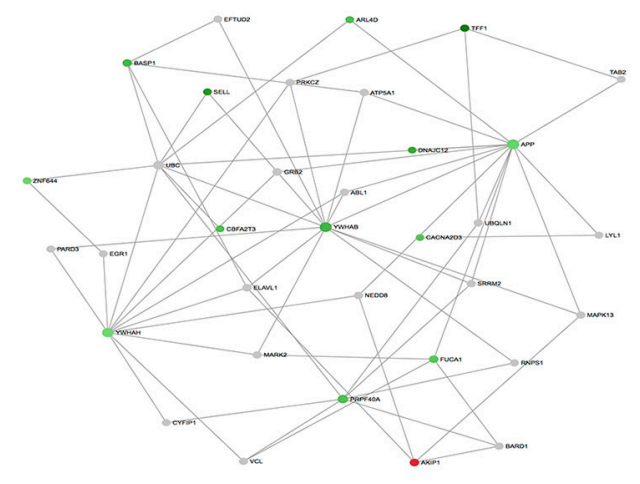

C
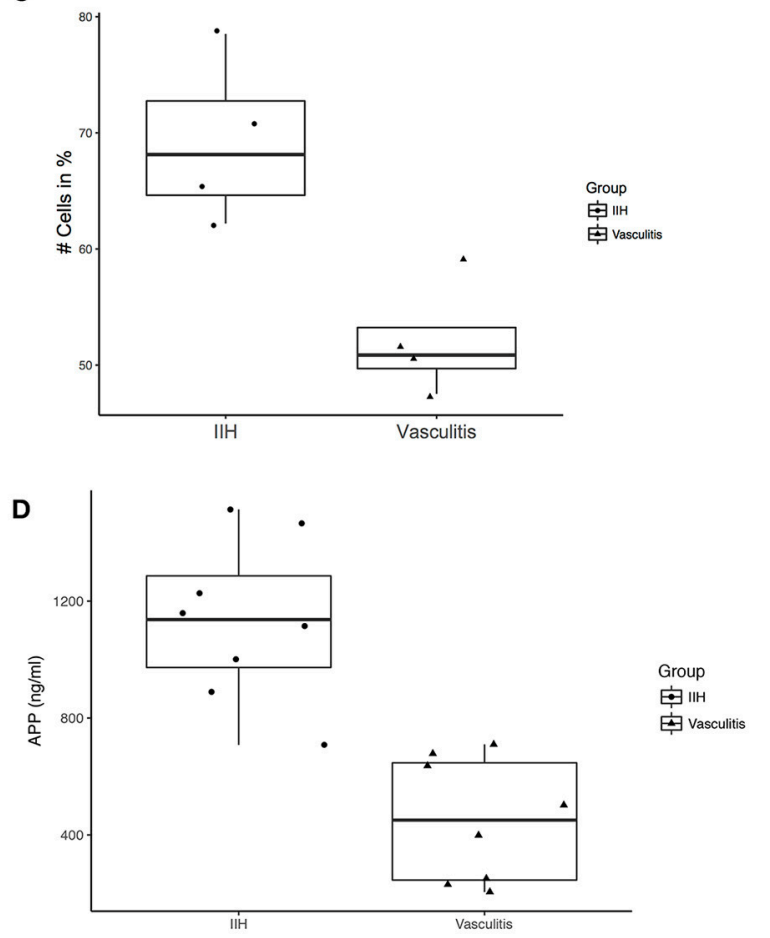

FIGURE 2 | Unbiased proteomics identifies proteins with differential abundance in CSF in PACNS. (A) Volcano plot showing all identified proteins and highlighting proteins with differential abundance. Proteins with log2(fold change) $>1$ are highlighted in orange and proteins with an adjusted $p$-value $\leq 0.05$ and a log2(fold change) $>1$ are highlighted in magenta comparing PACNS vs. IIH samples. Names of proteins with significantly different abundance are provided in the figure. (B) Network of proteins with an adjusted $p$-value $\leq 0.05$ built with NetworkAnalyst. The network shows the proteins

(Continued)
FIGURE 2 | identified in our LC-UDMSe analysis as well as proteins that directly interact with a given protein to study key nodes of functional connectivity. Red color indicates upregulated, green color downregulated proteins based on fold change. (C) Boxplot of the percentage of CD3+ T cells in peripheral blood for PACNS vs. IIH samples $(p=0.029)$. (D) Boxplot of the concentrations of APP in CSF of PACNS vs. $1 \mathrm{HH}$ samples ( $p=6.41 \mathrm{E}-05)$.

evidence regarding the relationship of proteins. Some of this evidence includes physical PPI but does not prove interaction of proteins in a specific cell type (e.g., immune cells). By depicting a PPI network we rather aimed to put the identified proteins into cellular and functional context to search for important nodes (hubs), which might be useful as biomarkers (30). We identified the downregulated amyloid beta A4 protein (gene APP) [adjusted $p$-value $<0.05, \log 2$ fold change (FC) $=-2.59$ ] as an important hub having several core connections in the newly defined network (Figure 2B). The network consisted of identified proteins as well as essential not detected proteins that maintained the network connection. Other notable candidate connections included Trefoil factor 1 (gene TFF1) (adj. $p<0.05$, $\log 2 \mathrm{FC}=-11.14), \mathrm{CD} 62 \mathrm{~L} / \mathrm{L}-$ selectin $($ gene $S E L L)($ adj. $p<0.05$, $\log 2 \mathrm{FC}=-8.26)$, brain acid soluble protein 1 (gene BASP1) $($ adj. $p<0.05, \log 2 \mathrm{FC}=-5.25), 14-3-3$ protein beta/alpha (gene $Y W H A B)($ adj. $p<0.05, \log 2 \mathrm{FC}=-5.49)$ and 14-3-3 protein eta (gene $Y W H A H)($ adj. $p<0.05, \log 2 \mathrm{FC}=-2.04)$. Therefore, changes of the CSF proteome in PACNS might reflect secondary nervous system pathology rather than immune activation. Nevertheless, these changes differ distinctly from findings in neurodegenerative disease $(28,31)$.

\section{DISCUSSION}

The objective of this study was to generate novel hypotheses regarding the molecular mechanisms driving PACNS-a rare but particularly disabling neurological disorder. We describe the first CSF-focussed unbiased proteomics screening approach to study local immunity in the vicinity of the CNS. In fact, we identified a number of proteins with differential abundance in PACNS (Figure 2A, Supplementary Table S1). Interesting candidates were APP and TFF1, SELL, BASP1, YWHAB, and YWHAH. Furthermore, APP could be validated using an additional protein quantification method in an extended cohort of patients supporting our technical approach and the potential relevance of APP in the pathogenesis of PACNS. All these proteins and the subsequent network analysis suggested that CSF in PACNS may reflect nervous system pathology rather than immune activation as significantly changed protein abundances were linked to neuronal rather than immune cell function. Based on these findings, we speculate that CSF markers of neuronal injury could be a surprisingly promising surrogate marker of CNS injury in PACNS if confirmed in subsequent studies. This study thus achieved its primary goal of articulating novel hypotheses in PACNS.

We performed down-stream analysis building a PPI network based on published evidence. This emphasized APP as central interaction hub and therefore potential key player in PACNS 
pathology. APP is one of the central proteins involved in CNS pathology in Alzheimer's disease (AD) (32). APP accumulates in extracellular plaques in $\mathrm{AD}$ and is cleaved by different secretases, which is thought to cause neuronal damage by disturbing signaling and inducing synaptotoxicity (33).

Studies concerning APP levels in AD have been inconsistent (34). A role of APP and its cleavage product beta-amyloid in the pathogenesis of other types of vasculopathy of the CNS has been reported previously (35). This has led to the proposal of a new subtype of CNS vasculopathy that has been named ABRA. Histopathologically ABRA was characterized by the presence of severe leptomeningeal and parenchymal amyloid angiopathy (36). Compared to our cohort with a mean age of 43 years, patients with ABRA in the above-mentioned study were generally older with a mean age of 67.3 years.

Downregulation of TFF1, which has been proposed as an anti-inflammatory agent in the brain, and downregulation of soluble L-Selectin having anti-inflammatory properties may reflect altered immune response in PACNS $(37,38)$. BASP1 has amyloid like properties for example by eliciting ion channels into membranes and is able to form fibril-like aggregates $(39,40)$. 14-3-3 proteins, on the other hand, control different cellular processes e.g., apoptosis. Deletion of YWHAH leads to severe disturbance of neuronal migration (41). During inflammatory signaling, suppression of YWHAH controls the induction of Toll-like receptor driven early pro-inflammatory cytokine and Type I interferon production (42) Furthermore, in large vessel vasculitides antibodies to 14-3-3 proteins were found pointing toward a roll of this protein family in PACNS (43).

Previous studies had shown that $\mathrm{CD} 4^{+} \mathrm{T}$ cells in the CSF of a patient with ABRA were increased (4), but this aspect had not been explored in PACNS. In the present study, we found no apparent differences in CSF cell composition by flow cytometry, which could reflect different mechanisms in ABRA and PACNS or may be due to the limited sample size of our cohort. We only found the percentage of $\mathrm{CD}^{+} \mathrm{T}$ cells in the blood to be decreased in PACNS vs. IIH controls which could be due to immunosuppressive treatment. The local activation of immune cells previously observed in biopsy studies, may therefore be reflected in the CSF (3).

Our present approach has limitations, which should be accounted for when interpreting the results. According to Calabrese and Mallek CNS tissue biopsy specimen is recommended for definite diagnosis of PACNS $(1,7)$. However, CNS biopsy is not performed in all patients and focussing only on biopsy-confirmed cases would have considerably limited available patient numbers and could have biased our study. Our study included patients who had been diagnosed based on angiography and black-blood contrast-enhanced T1 MRI images to confirm vessel abnormalities indicative of vasculitis, as well as CSF analysis to rule out infectious disease (9-12). This limitation could potentially result in inadvertently including patients with non-PACNS-related vessel alterations. We do, however, consider this possibility unlikely because we thoroughly selected and screened patients for differential diagnoses and all patients were repeatedly seen in our department after sampling. This long-term follow-up most likely excluded non-PACNS cases in our cohort.

The non-hypothesis driven proteomics approach of our study comes with inherent weaknesses. Our study does not provide mechanistic explanations for the reduction of neuronal protein abundance in the CSF in PACNS. Instead it provides the basis for better understanding the disease in the future. The ability to articulate new hypotheses is the key strength of such biomarker discovery studies.

Our study implies that CSF represents a unique window to better understand local immune reactions and neurodegenerative mechanisms in PACNS. If our findings were verified in larger cohorts and by additional techniques, it would allow confirming our list of candidates as potential diagnostic biomarkers. Future studies could also be designed to test for the prognostic values of our candidates. In conclusion, our findings suggest that APP and its possible interaction partners might be interesting candidate biomarkers to further investigate in patients with PACNS and that CSF could further help to diagnose PACNS in the future.

\section{ETHICS STATEMENT}

This study was carried out in accordance with the recommendations of the ethics committee of the medical association Westphalia-Lippe, Germany with written informed consent from all subjects. All subjects gave written informed consent in accordance with the Declaration of Helsinki. The protocol was approved by the ethics committee of the medical association Westphalia-Lippe, Germany (2016-053-f-S).

\section{AUTHOR CONTRIBUTIONS}

TR and GMzH designed the study, recruited patients, performed statistical analyses, and wrote the manuscript. JW performed the ELISA and data analysis. SK performed the LC-UDMSe work including bioinformatic data analysis using Progenesis QIP. AS-M and CG performed the FACS work. TR and MG did the network as well as additional statistical analyses of the proteomic data. GMzH, SM, and HW supervised the study and were involved in its design. All authors contributed to and have approved the final manuscript.

\section{ACKNOWLEDGMENTS}

This study was funded in part by grants from the Ministerium für Innovation, Wissenschaft und Forschung des Landes Nordrhein-Westfalen (MIWF NRW), and by project grants from the Deutsche Forschungsgemeinschaft (DFG grant ME4050/41), from the Hertie foundation and from the Innovative Medical Research (IMF) program of the Westfälische WilhelmsUniversity Münster (all to GMzH). The authors thank A.M. Berg for expert technical assistance and Antje Echterhoff for sample management. 


\section{SUPPLEMENTARY MATERIAL}

The Supplementary Material for this article can be found online at: https://www.frontiersin.org/articles/10.3389/fneur. 2018.00407/full\#supplementary-material

Supplementary Table S1 | List of all identified proteins using ROTS.

\section{REFERENCES}

1. Berlit P. Diagnosis and treatment of cerebral vasculitis. Ther Adv Neurol Disord. (2010) 3:29-42. doi: 10.1177/1756285609347123

2. Hajj-Ali RA, Singhal AB, Benseler S, Molloy E, Calabrese LH. Primary angiitis of the CNS. Lancet Neurol. (2011) 10:561-72. doi: 10.1016/S1474-4422(11)70081-3

3. Miller DV, Salvarani C, Hunder GG, Brown RD, Parisi JE, Christianson TJ, et al. Biopsy findings in primary angiitis of the central nervous system. Am J Surg Pathol. (2009) 33:35-43. doi: 10.1097/PAS.0b013e318181e097

4. Melzer N, Harder A, Gross CC, Wölfer J, Stummer W, Niederstadt T, et al. $\mathrm{CD} 4+\mathrm{T}$ cells predominate in cerebrospinal fluid and leptomeningeal and parenchymal infiltrates in cerebral amyloid $\beta$-related angiitis. Arch Neurol. (2012) 69:773-7. doi: 10.1001/archneurol.2011.2441

5. Thom V, Schmid S, Gelderblom M, Hackbusch R, Kolster M, Schuster $\mathrm{S}$, et al. IL-17 production by CSF lymphocytes as a biomarker for cerebral vasculitis. Neurol Neuroimmunol Neuroinflammation (2016) 3:e214. doi: 10.1212/NXI.0000000000000214

6. Bogner S, Bernreuther C, Matschke J, Barrera-Ocampo A, Sepulveda-Falla D, Leypoldt $\mathrm{F}$, et al. Immune activation in amyloid-beta-related angiitis correlates with decreased parenchymal amyloid-beta plaque load. Neurodegener Dis. (2014) 13:38-44. doi: 10.1159/000352020

7. Calabrese LH, Mallek JA. Primary angiitis of the central nervous system. Report of 8 new cases, review of the literature, and proposal for diagnostic criteria. Medicine (1988) 67:20-39. doi: 10.1161/STROKEAHA.116.013874

8. Birnbaum J, Hellmann H. Primary angiitis of the central nervous system. Arch Neurol. (2009) 66:704-709. doi: 10.1001/archneurol.2009.76

9. Saam T, Habs M, Pollatos O, Cyran C, Pfefferkorn T, Dichgans M, et al. High-resolution black-blood contrast-enhanced T1 weighted images for the diagnosis and follow-up of intracranial arteritis. Br. J. Radiol. (2010) 83:e1824. doi: 10.1259/bjr/74101656

10. Noh HJ, Choi JW, Kim JP, Moon GJ, Bang OY. Role of highresolution magnetic resonance imaging in the diagnosis of primary angiitis of the central nervous system. J Clin Neurol. (2014) 10:267-71. doi: $10.3988 /$ jen.2014.10.3.267

11. Obusez EC, Hui F, Hajj-Ali RA, Cerejo R, Calabrese LH, Hammad T, et al. High-resolution MRI vessel wall imaging: spatial and temporal patterns of reversible cerebral vasoconstriction syndrome and central nervous system vasculitis. AJNR Am J Neuroradiol. (2014) 5:1527-32. doi: 10.3174/ajnr.A3909

12. Mandell DM, Matouk CC, Farb RI, Krings T, Agid R, Terbrugge K, et al. Vessel wall MRI to differentiate between reversible cerebral vasoconstriction syndrome and central nervous system vasculitis: preliminary results. Stroke (2012) 43:860-2. doi: 10.1161/STROKEAHA.111.626184

13. Juneja M, Azmi A, Baets J, Roos A, Jennings MJ, Saveri P, et al. PFN2 and GAMT as common molecular determinants of axonal Charcot-Marie-Tooth disease. J Neurol Neurosurg Psychiatry (2018). doi: 10.1136/jnnp-2017-317562. [Epub ahead of print].

14. Zhu Q, Wu N, Liu G, Zhou Y, Liu S, Chen J, et al. Comparative analysis of serum proteome in congenital scoliosis patients with TBX6 haploinsufficiency - a first report pointing to lipid metabolism. J Cell Mol Med. (2018) 22:533-45. doi: $10.1111 / \mathrm{jcmm} .13341$

15. Hathout Y, Brody E, Clemens PR, Cripe L, DeLisle RK, Furlong P, et al. Largescale serum protein biomarker discovery in Duchenne muscular dystrophy. Proc Natl Acad Sci USA. (2015) 112:7153-8. doi: 10.1073/pnas.1507719112

16. Bot M, Chan MK, Jansen R, Lamers F, Vogelzangs N, Steiner J, et al. Serum proteomic profiling of major depressive disorder. Transl Psychiatry (2015) 5:e599. doi: 10.1038/tp.2015.88
Supplementary Table S2 | Results of the flow cytometry of CSF and blood expressed as percentage of cells.

Supplementary Tables S3, S4 | Patient demographics and clinical characteristics of the additional four PACNS and four $\mathrm{IH}$ patients. (for details see additional document).

Supplementary Table S5 | Results of the APP ELISA. Samples were run in duplicate.

17. Surinova S, Radová L, Choi M, Srovnal J, Brenner H, Vitek O, et al. Noninvasive prognostic protein biomarker signatures associated with colorectal cancer. EMBO Mol Med. (2015) 7:1153-65. doi: 10.15252/emmm

18. Distler U, Kuharev J, Navarro P, Tenzer S. Label-free quantification in ion mobility-enhanced data-independent acquisition proteomics. Nat Protoc (2016) 11:795-812. doi: 10.1038/nprot.2016.042

19. Lueg G, Gross CC, Lohmann H, Johnen A, Kemmling A, Deppe M, et al Clinical relevance of specific T-cell activation in the blood and cerebrospinal fluid of patients with mild Alzheimer's disease. Neurobiol Aging (2015) 36:81-9. doi: 10.1016/j.neurobiolaging.2014.08.008

20. R Development Core Team. R: A Language and Environment for Statistical Computing. R Foundation statistical computing Vienna (2013).

21. Elo LL, Filén S, Lahesmaa R, Aittokallio T. Reproducibility-optimized test statistic for ranking genes in microarray studies. In: IEEE/ACM Transactions on Computational Biology and Bioinformatics, (Los Alamitos, CA), p. 423-31.

22. Suomi T, Seyednasrollah F, Jaakkola MK, Faux T, Elo LL. ROTS: an R package for reproducibility-optimized statistical testing. PLoS Comput Biol. (2017) 13:e1005562. doi: 10.1371/journal.pcbi.1005562

23. Mann M, Wilm M. Error-tolerant identification of peptides in sequence databases by peptide sequence tags. Anal Chem. (1994) 66:4390-9. doi: 10.1021/ac00096a002

24. Breuer K, Foroushani AK, Laird MR, Chen C, Sribnaia A, Lo R, et al. InnateDB: systems biology of innate immunity and beyond - Recent updates and continuing curation. Nucleic Acids Res. (2013) 41:D1228-33. doi: $10.1093 /$ nar/gks1147

25. Szklarczyk D, Franceschini A, Wyder S, Forslund K, Heller D, Huerta-Cepas J, et al. STRING v10: Protein-protein interaction networks, integrated over the tree of life. Nucleic Acids Res. (2015) 43:D447-52. doi: 10.1093/nar/gku1003

26. Xia J, Gill EE, Hancock REW. NetworkAnalyst for statistical, visual and network-based meta-analysis of gene expression data. Nat Protoc. (2015) 10:823-44. doi: 10.1038/nprot.2015.052

27. Ottervald J, Franzén B, Nilsson K, Andersson LI, Khademi M, Eriksson B, et al. Multiple sclerosis: Identification and clinical evaluation of novel CSF biomarkers. J Proteomics (2010) 73:1117-32. doi: 10.1016/j.jprot.2010.01.004

28. Wildsmith KR, Schauer SP, Smith AM, Arnott D, Zhu Y, Haznedar J, et al. Identification of longitudinally dynamic biomarkers in Alzheimer's disease cerebrospinal fluid by targeted proteomics. Mol Neurodegener. (2014) 9:22. doi: 10.1186/1750-1326-9-22

29. Dayon L, Turck N, Garci-Berrocoso T, Walter N, Burkhard PR, Vilalta A, et al. Brain extracellular fluid protein changes in acute stroke patients. J Proteome Res. (2011) 10:1043-51. doi: 10.1021/pr101123t

30. Xia J, Benner MJ, Hancock REW. NetworkAnalyst-integrative approaches for protein-protein interaction network analysis and visual exploration. Nucleic Acids Res. (2014) 42:W167-74. doi: 10.1093/nar/gku443

31. Halbgebauer S, Öckl P, Wirth K, Steinacker P, Otto M. Protein biomarkers in Parkinson's disease: focus on cerebrospinal fluid markers and synaptic proteins. Mov Disord. (2016) 31:848-60. doi: 10.1002/mds.26635

32. O'Brien RJ, Wong PC. Amyloid precursor protein processing and Alzheimer's Disease. Annu Rev Neurosci. (2011) 34:185-204. doi: 10.1146/annurev-neuro-061010-113613

33. Andrew RJ, Kellett KAB, Thinakaran G, Hooper NM. A Greek tragedy: the growing complexity of Alzheimer amyloid precursor protein proteolysis. J Biol Chem. (2016) 291:19235-44. doi: 10.1074/jbc.R116.746032

34. Craig-Schapiro R, Fagan AM, Holtzman DM. Biomarkers of Alzheimer's disease. Neurobiol Dis. (2009) 35:128-40. doi: 10.1016/j.nbd.2008.10.003

35. Smith EE, Greenberg SM. Beta-amyloid, blood vessels, and brain function. Stroke (2009) 40:2601-6. doi: 10.1161/STROKEAHA.108.536839 
36. Scolding NJ, Joseph F, Kirby PA, Mazanti I, Gray F, Mikol J, et al. Abetarelated angiitis: primary angiitis of the central nervous system associated with cerebral amyloid angiopathy. Brain (2005) 128:500-15. doi: 10.1093/brain/ awh379

37. Znalesniak EB, Fu T, Guttek K, Händel U, Reinhold D, Hoffmann W. Increased cerebral Tff1 expression in two murine models of neuroinflammation. Cell Physiol Biochem. (2016) 39:2287-96. doi: 10.1159/000447921

38. Smalley DM, Ley K. L-selectin: mechanisms and physiological significance of ectodomain cleavage. J Cell Mol Med. (2005) 9:255-66. doi: 10.1111/j.1582-4934.2005.tb00354.x

39. Ostroumova OS, Schagina LV, Mosevitsky MI, Zakharov VV. Ion channel activity of brain abundant protein BASP1 in planar lipid bilayers. FEBS J. (2011) 278:461-9. doi: 10.1111/j.1742-4658.2010.07967.x

40. Zakharov VV, Mosevitsky MI. Oligomeric structure of brain abundant proteins GAP-43 and BASP1. J Struct Biol. (2010) 170:470-83. doi: $10.1016 /$ j.jsb.2010.01.010

41. Berg D, Holzmann C, Riess O. 14-3-3 proteins in the nervous system. Nat Rev Neurosci. (2003) 4:752-62. doi: 10.1038/nrn1197
42. Butt AQ, Ahmed S, Maratha A, Miggin SM. 14-3-3 \{epsilon\} and 14-3-3 $\sigma$ inhibit TLR-mediated pro-inflammatory cytokine induction. J Biol Chem. (2012) 87:38665-79. doi: 10.1074/jbc.M112.367490

43. Chakravarti R, Gupta K, Swain M, Willard B, Scholtz J, Svensson LG, et al. 143-3 in Thoracic aortic aneurysms: identification of a novel autoantigen in large vessel vasculitis. Arthr Rheumatol. (2015) 67:1913-21. doi: 10.1002/art.39130

Conflict of Interest Statement: The authors declare that the research was conducted in the absence of any commercial or financial relationships that could be construed as a potential conflict of interest.

Copyright (c) 2018 Ruland, Wolbert, Gottschalk, König, Schulte-Mecklenbeck, Minnerup, Meuth, Groß, Wiendl and Meyer zu Hörste. This is an open-access article distributed under the terms of the Creative Commons Attribution License (CC $B Y)$. The use, distribution or reproduction in other forums is permitted, provided the original author(s) and the copyright owner are credited and that the original publication in this journal is cited, in accordance with accepted academic practice. No use, distribution or reproduction is permitted which does not comply with these terms. 